\title{
The Effects of Marital Conflicts Perceived by Adolescent Children on Bullying: The Dual Mediating Effects of Self-System Traumatization and Violence Tolerance
}

\author{
Su Jeong Kim ${ }^{1}$ Jung Min $\mathrm{Kim}^{2}$, In Hee Hwang ${ }^{3}$ \\ M. A., Department of Child Development and Education, Myongji University, Seoul, Korea ${ }^{1}$ \\ Professor, Department of Child Development and Education, Myongji University, Seoul, Korea ${ }^{2}$ \\ M. A. Student, Department of Child Development and Education, Myongji University, Seoul, Korea ${ }^{3}$ \\ 청소년 자녀가 지각한 부부갈등이 또래괴롭힘에 미치는 영향 : \\ 자기체계손상과 폭력허용도의 이중매개효과 \\ 김수정 ${ }^{1}$, 김정민 ${ }^{2}$, 황인희 ${ }^{3}$ \\ 명지대학교 아동학과 석사, 명지대학교 아동학과 교술, 명지대학교 아동학과 석사과정
}

Objectives: This study's purpose was to examine the effects of marital conflicts perceived by adolescents on self-system traumatization, violence tolerance, and bullying. Additionally, the dual mediating effects of self-system traumatization and violence tolerance on the relationship between marital conflicts perceived by adolescent children and bullying were examined.

Methods: Participants were middle and high school students in Seoul and Gyeonggi-do, who had experienced bullying. Data analysis was conducted through descriptive statistics using SPSS Statistics 21.0 and the separate and dual mediating effect analyses were conducted using SPSS Marco.

Results: Both self-system traumatization and violence tolerance had a partial mediation effect on the relationship between marital conflicts perceived by adolescent children and bullying. Moreover, selfsystem traumatization and violence tolerance had a full dual-mediation effect on the relationship between marital conflict perceived by adolescent children and bullying.

Conclusion: The results indicate to provide preventive data on adolescents who harassed their peers by looking at the dual mediating effects of self-system traumatization and violence tolerance based on the relationship between marital conflict and bullying.

Keywords: conflicts perceived by adolescent children, bullying, self-system traumatization, violence tolerance, adolescent

\section{Introduction}

또래괴롭힘(Bullying)이란 또래 간 힘의 불평등 관계에서 일 어나는 현상으로 힘이 강한 집단이나 개인이 힘이 약한 개인

Corresponding Author: Jung Min Kim, Professor, Department of Child Development and Education, Myongji University, Seoul, Korea E-mail: jmkim122@mju.ac.kr
에게 지속적이고 의도적으로 공격을 가하는 행동을 의미한다 (Olweus, 1993). 또래괴롭힘은 크게 폭행 · 구타와 같은 신체적 괴롭힘과 욕설·소문 퍼뜨리기 같은 언어적 괴롭힘, 무시 - 따 돌림과 같은 소외로 나누어질 수 있다(E. S. Choi, 2000). 신체

(C)The Korean Association of Child Studies

This is an Open Access article distributed under the terms of the Creative Commons Attribution Non-Commercial License (http:// creativecommons.org/licenses/by-nc/4.0) which permits unrestricted noncommercial use, distribution, and reproduction in any medium, provided the original work is properly cited. 
적 괴롭힘은 의도적으로 신체에 해를 끼치는 괴롭힘을 의미하 며, 언어적 괴롭힘은 욕설이나 피해 대상에 대한 안 좋은 소문 을 내는 것으로 신체적 괴롭힘보다는 간접적인 방법을 의미한 다. 소외란, 같이 어울리는 무리에 끼워주지 않는 의도적인 따 돌림을 의미하며 언어적 괴롭힘과 같은 간접적인 방법에 속 한다(C. J. Lee \& Kwak, 2000). 또한 학교폭력에 관한 법률에서 는 또래괴롭힘의 장소를 학교 내외로, 대상은 학생으로 한정 하고 있으며, 공격 행위의 범위는 상해·폭행뿐 아니라 언어 적 폭력 및 성폭력, 인터넷 - 정보통신기기를 이용한 사이버 폭 력까지 확대 규정하였다(학교폭력예방 및 대책에 관한 법률, 2012.03.21. 제2조). 이렇듯 또래괴롭힘은 매일 만나게 되는 학 교생활권에서 발생하기 때문에 피해자에게 큰 고통을 준다는 부분에서 심각한 문제행동으로 여겨진다(Olweus, 1978; Perry, Kusel, \& Perry, 1988).

청소년기는 부모로부터 독립해 또래 관계를 보다 중요하게 생각하는 시기이다(C. H. Moon, Park, Jeon, \& Cheon, 2010). 청소년기의 만족스럽고 긍정적인 또래 관계는 청소년의 긍정 적 자아 정체감 형성, 정서적 안정, 소속감 경험 등 청소년의 전반적 발달에 기여한다(Collins \& Laursen, 2004; Yoon, Lee, \& Min, 2012). 그러나 부정적인 또래 관계는 청소년의 낮은 자존 감과 정서적 불안정감, 정체감 혼란, 사회적 기술 발달 저해 등 청소년기 전반적 발달에 부정적 영향을 끼치는 것으로 알려져 있다(Olweus, 1993; Seol, Kyeong, \& Ji, 2015). 따라서 또래 괴롭 힘은 청소년에게 중요한 문제라고 할 수 있다.

최근 교육부가 주관하여 실시한 “ 18 년도 제 1 차 학교폭력 실태조사" 결과에 따르면 “또래괴롭힘 피해를 당한 적이 있 다.”고 응답한 학생은 참여 학생의 $1.7 \%$ 로 2017년 1차 대비 $0.6 \%$ 가 증가했다(Ministry of Education, 2018). 또한 학교폭력 집단가해 학생 수는 2015년 대비 2016년에는 중학생 가해자 증가율이 $119 \%$, 고등학생 가해자 증가율이 $121 \%$ 를 보이며 가해학생의 수가 급격한 증가 추이를 보이고 있다(I. S. Kim, 2018). 선행연구에 따르면 또래괴롭힘은 피해학생은 물론이 고 가해학생에게도 심리 - 정서 - 사회적 문제를 일으킨다고 알 려져 있다(K. Kim, Lee, \& Shin, 2013; M. K. Oh, Ahn, \& Kim, 2013). 또래괴롭힘을 경험한 피해학생은 불안, 우울, 낮은 자 존감, 공격성, 자살사고 등 내적인 고통을 경험한다(Hanish \& Guerra, 2002; H. Kim, 2013; J. K. Kwon, 2011; Swearer, Song, Cary, Eagle, \& Mickelson, 2001). 뿐만 아니라 뇌발달 이상, 학 업중단, 보복성 폭력, 자살에 이르는 외현화된 문제들로까지 나타나고 있다(Gini \& Espelage, 2014; J. Y. Kim, Jang, \& Min, 2011; Ozer \& Weinstein, 2004; H. S. Song, 2014). 또래괴롭힘
가해 학생 역시 불안과 우울 분노 등 심리 - 사회적 어려움을 경험하게 되는데(K. Kim et al., 2013; M. K. Oh et al., 2013), 이 와 같은 어려움이 방치되면 품행장애, 반항성 장애, 낮은 지능, 과잉행동 장애 등의 문제를 가질 수 있고, 반사회적 경향과 높 은 일탈행동의 경향으로 발전될 수 있다(Baldry \& Farrington, 2000; Y.-L. Moon, Choi, Paek, \& Kim, 2007). 또래괴롭힘 가해 에 대한 근본적인 개입이 없다면 또래괴롭힘 피해 청소년에 대한 폭력이 지속될 수밖에 없으며 폭력에 대한 무감각, 청소 년 범죄와 같은 다양한 심리적 · 정서적 · 행동적 문제에 노출 된 가해 청소년들은 건강한 사회 구성원으로 성장할 수 없게 된다(Baldry \& Farrington, 2000; S. Y. Lee \& Jung, 2015).

청소년들의 또래 관계 및 문제행동에 영향을 미치는 주요 한 환경적 요인으로는 청소년이 인식한 부모의 부부갈등을 들 수 있다(Buehler \& Gerard, 2002; Fincham, Grych, \& Osborne, 1994; S. Y. Lee \& Jung, 2015; Yoon et al., 2012). 부부갈등이란 부부가 서로의 불일치와 대립을 해결하기 위해 교환하게 되는 부정적인 언어와 신체적 싸움 등을 포함한다(J. A. Jang \& Shin, 2006). 부부갈등은 자녀의 연령이나 성별에 상관없이 자녀에 게 부정적 영향을 미친다(Buehler \& Gerard, 2002; E.-J. Song \& Lee, 2010). 특히 청소년기는 부모에서 또래로 애착관계가 이 동하는 동시에 부모가 주요한 안전기지로 작용하는 시기이기 때문에 이 시기에 지각하는 부부갈등은 청소년의 적응에 중요 한 영향을 미칠 수 있다(Buchanan \& Waizenhofer, 2001; J.-H. Kim \& Lee, 2017; Min \& Choi, 2014). 선행연구에 따르면, 자 녀들이 부모의 분노에 찬 갈등을 목격한 후에 공격행동을 보 였으며(J. Cha, Lee, \& Hwang, 2009; Park \& Kang, 2017), 부부 갈등을 자주 경험한 자녀들은 내재화 혹은 외현화 문제를 일 으켰다(Y. S. Kim, Park, \& Kim, 2018; Park \& Kang, 2017). 이 처럼 가정에서 부모의 언어적 - 신체적 갈등을 많이 목격한 자 녀는 공격적이게 되기 쉽고(Hur \& Nam, 2016; S. I. Im \& Lee, 2013), 또래괴롭힘 가해를 일으키는 것으로 밝혀졌다(Boivin, Lavoie, Hébert, \& Gagne, 2012; Cho \& Cho, 2011; J. A. Oh, Kim, \& Yoon, 2018; Yoon et al., 2012).

한편, 본 연구에서는 청소년 자녀가 지각한 부부갈등과 또 래괴롭힘 가해와의 관계에서 자기체계손상에 대하여 살펴보 았다. 자기체계손상(self-system traumatization)은 대인 간 외상 으로 인하여 개인의 심층적인 성격 구조에 변화가 일어나는 것으로 주체적 자기손상, 대상적 자기손상, 자기조절손상, 관 계적 자기손상을 포함하는 개념이다(Cook et al., 2005; Harter, 1999; Van der Kolk, Roth, Pelcovitz, Sunday, \& Spinazzola, 2005). 주체적 자기(i-self) 손상은 “나는 누구인가"와 관련된 부분으 
로 자기자각, 자기주도성, 자기지속성, 자기통합성의 손상 등 을 포괄하는 개념이다. 대상적 자기(me-self) 손상은 다른 사람 과의 상호작용에서 형성되는 대상으로써의 자기가 와해되는 것을 의미한다. 자기조절(self-regulation)은 인간이 자신의 마음 의 기능과 상태 및 내적과정 등에 대해 통제력을 행사하는 과 정(Finkel \& Baumeister, 2010)으로 자기조절손상은 목표를 달 성하기 위해 자신의 사고, 정서 및 행동 등을 의식적으로 노력 하는 과정을 실패하는 것을 의미한다(Cook et al., 2005). 끝으 로 관계적 자기(relational self) 손상은 자기와 타인 그리고 대인 관계에 대한 표상이 왜곡된 상태를 의미한다(Salo, Punamaki, \& Qouta, 2004). 부모의 부부갈등을 목격한 청소년들은 중요 한 타인에게 존중받지 못하고 정서적 폭력을 경험하면서 부 정적 자기개념 혹은 낮은 자존감이 형성되고 자기증오, 자기 비난 등을 포함하는 부정적 자기감이 유발된다(Allen, 2001; Harter, 1999; A. Kim \& Lee, 2016; J. A. Oh \& Kim, 2015; Won $\&$ Gong, 2017). 또한, 부부갈등은 자녀의 자기조절에 부정적 인 영향을 미쳐 분노정서 경험수준이 높고 공격성을 포함하 는 외현화 문제행동에 영향을 미친다(Ahn \& Ahn, 2012; Y.-S. Kim, Park, \& Kim, 2018). 이러한 연구 결과는 대인 간 외상으 로 일어나는 자기체계손상이 어린 시절부터 지속적으로 경험 한 부모의 부부갈등으로 인하여 청소년들의 자기체계가 손상 될 것으로 예측할 수 있다. 즉, 부부갈등을 경험한 청소년은 건 강한 자기(self)를 형성하는데 어려움이 있고, 대인관계에서 공격적인 행동을 보일 것이다.

최근 또래괴롭힘을 예측하는 변인으로 폭력허용도가 다 루어지고 있다(J.-Y. Ahn, 2016; K.-E. Kim \& Yoon, 2012; K.H. Noh \& Hahn, 2017; K.-H. Noh \& Joo, 2015). 폭력허용도 (violence tolerance)란 관계 안에서 폭력에 대한 필요와 허용적 인식에 관한 지표로서 "폭력에 대해 얼마나 허용적으로 생각 하는가?”로 정의된다(J.-Y. Kim, Choi, \& Lim, 2015; S.-W. Lee $\& \mathrm{Jo}, 2005)$. 폭력에 대해 허용적으로 생각한다는 것은 인지적 태도 중 하나인데, 인지적 태도는 똑같은 사건에 대해서 개인 에 따라 다르게 인식하도록 하는 지표이다(K.-H. Noh \& Joo, 2015). 청소년의 폭력허용도와 관련된 선행연구를 살펴보면 폭력허용도가 높은 청소년은 갈등상황이나 문제해결에서 폭 력의 사용을 허용하고 폭력의 정당성과 필요성을 수용하기 때 문에 쉽게 폭력을 사용하는 것으로 나타났다(J.-Y. Ahn, 2016; H.-Y. Choi, 2011; K.-E. Kim \& Yoon, 2012; K.-H. Noh \& Hahn, 2017; J.-A. Oh, Park, \& Lim, 2016; Rigby \& Slee, 1991). 따라서 폭력허용도가 높은 청소년들은 또래괴롭힘 가해를 많이 일으 킬 것으로 예측할 수 있다. 특히, 부모요인은 자녀의 폭력허용
도에 직접적인 영향을 미쳤는데 부모의 양육 행동에 따라 폭 력허용도와 또래괴롭힘 가해에 차이가 나타났다(K.-H. Noh $\&$ Hahn, 2017; J.-A. Oh et al., 2016). 부모의 부부갈등상황에서 폭력을 자주 경험한 자녀들은 상대적으로 폭력에 대해 둔감 하고 이를 허용하는 태도를 지니게 되고(J.-I. Im \& Kim, 2010; Mun \& Chung, 2015), 폭력허용도가 높아지면 또래괴롭힘을 일으킬 것으로 예측할 수 있다. 부정적인 자기감의 대표적 정 서인 죄책감과 수치심은 공격성을 높이고(J.-W. Im \& Hong, 2016; Shin \& Lee, 2015), 공격성과 폭력허용도가 정적 상관이 있으므로(J.-I. Lee \& Kim, 2015; K.-H. Noh \& Hahn, 2017), 자 기체계가 손상 될수록 폭력허용도가 높아질 것이다. 따라서 자기체계손상과 그로인한 폭력허용도가 순차적으로 작용할 것임을 예측할 수 있다. 이러한 선행연구에 근거하여 자기체 계손상과 폭력허용도가 또래괴롭힘 가해에 어떠한 경로로 영 향을 미칠 것인지 살펴보고자 한다.

기존의 또래괴롭힘 연구는 부모의 양육태도, 가정폭력과 같은 가족요인에 대한 연구가 많이 이루어졌지만 부부갈등과 또래괴롭힘 가해에 대한 연구는 상대적으로 미비하다. 또래괴 롭힘은 또래와의 갈등상황에서 갈등을 어떻게 해결하느냐에 따라 발생가능성이 달라지기 때문에 자녀에게 가장 밀접한 환 경인 가정에서 부모의 부부갈등을 어떻게 지각하고 있는지가 또래괴롭힘 가해를 예측하는데 중요한 요인이 될 것이다. 또 한, 부부갈등과 자녀의 부적응에 대한 연구들은 주로 아동기 를 대상으로 하고 있어 초기청소년기인 중학생과 성인기를 앞 두고 있는 후기청소년기인 고등학생을 대상으로 한 연구는 미 비하므로 중·고등학생을 대상으로 또래괴롭힘 문제를 살펴보 고자 한다(J. Kim, 2011). 자녀가 지각한 부부갈등과 또래괴롭 힘 가해와의 관계에서 자기통제, 자존감, 자아개념과 같은 단 편적인 연구들이 아닌 총체적인 자기의 손상을 의미하는 자기 체계손상과의 연관성을 살펴봄으로써 부모의 부부갈등이라 는 대인외상으로 인해 개인의 심층적인 성격구조의 변화가 또 래괴롭힘 가해에 어떤 영향력을 미치는지 알아보는 것은 의미 가 있을 것이다. 특히, 폭력허용도를 통해 청소년들이 가지고 있는 폭력에 대한 인식을 알아봄으로써 심리 · 정서적인 요인 뿐만이 아니라 폭력에 대한 인식이 행위로 이어짐을 확인하고 폭력허용도가 또래괴롭힘을 일으키는 중요한 작용기제로 발 휘됨을 예측할 수 있다.

이에 본 연구는 또래괴롭힘 가해에 영향을 미치는 가족 변인 으로 자녀가 지각한 부부갈등, 자기내적 변인으로 자기체계손 상, 인지적 변인으로 폭력허용도를 선택하여 이들이 어떠한 경 로로 또래괴롭힘 가해에 이르게 되는지를 연구해보고자 한다. 


\section{연구문제 1}

청소년 자녀가 지각한 부부갈등이 또래괴롭힘 가해에 미치는 영향에 대한 자기체계손상과 폭력허용도의 개별 매개효과는 각각 어떠한가?

\section{연구문제 2}

청소년 자녀가 지각한 부부갈등이 또래괴롭힘 가해에 미치는 영향에 대한 자기체계손상과 또래괴롭힘의 이중매개 효과는 어떠한가?

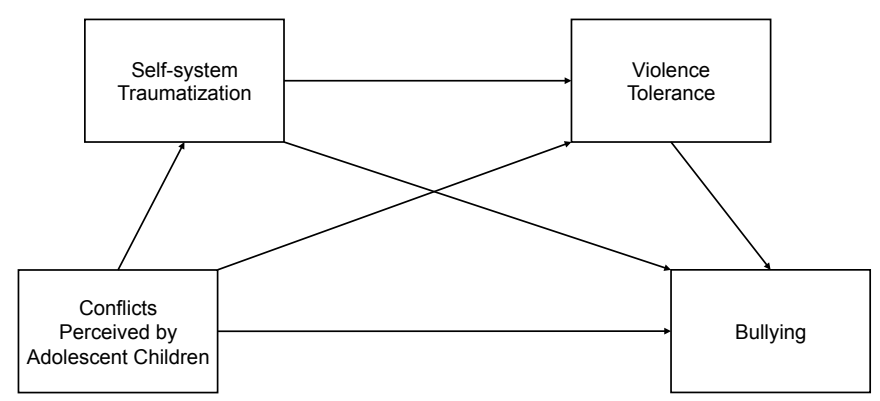

Figure 1. Study model.

\section{Methods}

\section{연구대상}

본 연구는 서울, 경기지역 소재 6개의 중.고등학교에 재학 중 인 남녀 청소년 총 550명을 대상으로 설문지를 실시하였다. 총 550 부의 질문지 중 불성실하게응답하거나 또래괴롭힘 가 해가 없다고 보고된 85 부를 제외한 465 부의 자료를 최종 연구 자료로 사용하였다. 연구대상의 성별 구분에 따르면 남자가 229 명(49.2\%), 여자가 236명(50.8\%)이었고, 학교 구분에 따르 면 중학생은 254 명(54.6\%), 고등학생은 211 명(45.4\%)이었다.

\section{연구도구}

\section{또래괴롭힘 가해}

학교폭력의 가해경험 정도를 알아보기 위해 E. S. Choi (2000) 가 제작한 집단따돌림 가해 검사 문항을 사용하였다. 본 척도 의 설문 구성의 하위요인은 소외형 5 문항, 언어형 5 문항, 신 체형 5 문항으로 구분되어 있으며 지난 일 년 동안의 학교폭력
가해 경험에 대해 조사한다. 집단따돌림 가해 척도는 각 문항 당 전혀 없었다는 0 점, 있었다에서 한 번 또는 두 번은 1 점, 가 끔은 2점, 일주일에 한 두 번씩은 3점, 일주일에 여러번은 4점 을 배점하여 따돌림 가해 0-4점의 점수범위를 가져, 점수가 높 을수록 집단따돌림 가해를 경험한 수준이 높음을 나타낸다. Likert식 5점 척도(전혀 없었다[0점] 일주일에 여러 번 있었 다[4점])로 평정된다. 본 연구에서 사용한 척도의 각 하위별 신 뢰도 계수는 소외형 .72, 언어형 .78, 신체형 .87이고 전체 신뢰 도 계수(Cronbach's $\alpha$ )는 .89로 나타났다.

\section{자녀가 지각한 부부갈등}

자녀가 지각한 부부갈등을 측정하기 위해 Grych, Seid와 Fincham (1992)가 개발한 Children's Perception of Interparental Conflict Scale (CPIC)를 Kwon과 Lee (1997)가 번안하여 타당 화한 것을 사용하였다. 본 척도는 44 개 문항으로 8 개의 하위 요인인 빈도 6 문항, 강도 7문항, 해결 6 문항, 내용 4 문항, 지각 된 위험 6 문항, 대처효율성 6 문항, 자기비난 5문항, 삼각관계 4문항으로 구성된다. 각 문항은 Likert식 5점 척도(전혀 그렇 지 않다[1점] 매우 그렇다[5점])로 평정된다. 평정된 점수는 최하 44점에서 최고 220점으로 점수가 높을수록 자녀가 지각 한 부부갈등이 높음을 의미한다. 긍정 응답 문항 $(1,2,5,8,12$, $1720,24,25,32,35,43$ 번 문항)에 대해서는 역채점을 하였다. 본 연구에서 사용한 척도의 각 하위별 신뢰도 계수는 빈도 .77, 강도 .84 , 해결 .83 , 지각된 위험 .86 , 대처효율성 .72 , 삼각관계 .67 , 자기비난 .73, 내용 .79이고 전체 신뢰도 계수(Cronbach's $\alpha)$ 는 .94로 나타났다.

\section{자기체계손상}

자기체계손상을 측정하기 위해 J. Jang (2010)가 개발한 자기 체계손상척도(Traumatic-Self-System Scale [TSSS])를 사용하 였다. 본 척도는 58 문항으로 주체적 자기손상, 대상적 자기손 상, 자기조절 손상, 관계적 자기손상의 4 개 하위 척도들로 구 성되어 있다. 각 문항은 Likert 5점 척도로 평정되며(전혀 그렇 지 않다[0점] 매우 그렇다[4점])로 평정된다. 평정된 점수는 최하 0점에서 최고 232점으로 점수가 높을수록 자기체계손 상정도가 높음을 의미한다. 본 연구에서 전체 척도의 신뢰도 (Cronbach's $\alpha$ )값은 .96이고 각 하위별 신뢰도 계수는 주체적 자기손상 .92 , 대상적 자기손상 .92 , 자기조절 손상 .83 , 관계적 자기손상 .75로 나타났다. 
Table 1

Correlation and Descriptive Statistics

\begin{tabular}{|c|c|c|c|c|}
\hline Variables & 1 & 2 & 3 & 4 \\
\hline Conflicts perceived by adolescent children & - & & & \\
\hline Self-system traumatization & $.41^{* * *}$ & - & & \\
\hline Violence tolerance & $.33^{* * *}$ & $.26^{* * *}$ & - & \\
\hline Bullying & $.25^{* * *}$ & $.34^{* * *}$ & $.52^{* * *}$ & 一 \\
\hline$M(S D)$ & $97.51(23.63)$ & $83.03(37.19)$ & $25.03(5.79)$ & $11.35(8.97)$ \\
\hline Skewness & -.05 & .04 & .59 & 1.30 \\
\hline
\end{tabular}

Note. $N=465.1$ = conflicts perceived by adolescent children; 2 = self-system traumatization; 3 = violence tolerance; $4=$ bullying. *** $p<.001$.

\section{폭력허용도}

폭력허용도를 측정하기 위하여 Rigby와 Slee (1991)의 척도와 Perry, Perry와 Ramussen (1986)의 척도를 Y.-S. Kim (2000)이 번 안하고 H.-Y. Choi (2011)이 재구성한 또래폭력에 대한 허용도 척도를 사용하였다. 본 척도는 14 개의 문항으로 2 개의 하위요 인 허용적 태도 9문항, 행위가능성 5 문항으로 구성되며 각 문 항은 Likert식 4점 척도(전혀그렇지 않다[1점], 매우 그렇다[4 점])로 평정된다. 평정된 점수는 최하 14점에서 최고 56점으로 점수가 높을수록 또래폭력에 대해 허용적인 태도를 가지는 것 으로 측정되고 긍정 응답 문항 $(4,5,6$ 번 문항)에 대해서는 역 채점을 하였다. 본 연구에서 사용한 척도의 전체 신뢰도 계수 (Cronbach's $\alpha$ )는 80 이었으며 각 하위별 신뢰도 계수는 허용 적 태도.67, 행위가능성 .79로 나타났다.

\section{연구절차}

서울, 경기지역 소재 6 개의 중 · 고등학교에 재학 중인 남녀 청 소년 총 550명을 대상으로 2018년 7월 2일부터 8월 31일까지 설문조사를 실시하였다. 질문지는 연구자가 연구의 목적과 비 밀보장에 대한 설명 후 배부하여 연구 참여에 대한 동의를 받 아 실시하였으며 담임교사의 안내 후 배부, 회수되었다. 질문 지 작성에는 총 20 여분이 소요되었다. 총 550 부의 질문지 중 불성실하게 응답하거나 또래괴롭힘 가해 행동을 한 적이 없 다고 보고한 85 부를 제외한 465 부의 자료를 최종 연구 자료로 사용하였다.

\section{자료분석}

수집된 자료는 SPSS Win 21.0 (IBM Co., Armonk, NY)과 SPSS
Macro (IBM Co., Armonk, NY) 통계 프로그램을 통해 분석 되었다. 척도의 신뢰도 측정을 위해 내적 일관성을 나타내는 Cronbach's $\alpha$ 값을 산출했으며, 기술통계 분석, $t$-검정, Pearson 의 상관관계분석 등을 실시하였다. 또한 Hayes (2013)가 제안 한 SPSS Macro를 사용한 매개효과 분석을 실시하였으며, 매개 효과의 유의성 검증을 위해 부트스트래핑(bootstrapping)을 사 용하였다. 자녀가 지각한 부부갈등과 또래괴롭힘 가해와의 관 계에서 자기체계손상과 폭력허용도의 역할을 확인하기 위하 여 SPSS Process Macro model 4를 사용하여 매개분석 하였고, 간접 효과의 유의성을 검증하기 위해 model 6 을 사용하여 순 차적 매개모형으로 분석하였다. 대상의 성별과 학년에 따라 주요변인에 차이가 있는지를 살펴보기 위해 독립표본 t-검정 을 실시하였을 때 종속변수인 또래괴롭힘 가해에 유의한 차이 를 나타낸 성별만 통제하여 실시하였다.

\section{Results}

\section{변인들의 기술적 통계}

먼저 각 변인들의 일반적 특성 및 정규성 분포를 확인하기 위 한 기술통계분석과 변인의 상관관계분석을 실시하였다. 분 석 결과는 Table 1 과 같다. 기술통계분석 결과, 각 변인의 왜 도(Skewness) 및 첨도(Kurtosis)의 절대값이 각각 3 과 10 을 넘 지 않아 정규성에는 문제가 없는 것으로 확인되었다(Kline, 2010). 상관관계분석 결과, 청소년이 지각한 부부갈등은 또래 괴롭힘 가해 $(r=.25, p<.001)$, 폭력허용도 $(r=.33, p<.001)$, 자 기체계손상 $(r=.41, p<.001)$ 과 각각 유의한 정적상관을 보였 다. 또한 자기체계손상은 또래괴롭힘 가해 $(r=.34, p<.001)$, 폭력허용도 $(r=.26, p<.001)$ 와 유의한 정적상관을 나타냈다. 
Table 2

Mediating Effects of Self-System Traumatization in the Relationship Between Conflicts Perceived by Adolescent Children and Bullying

\begin{tabular}{lcccccc}
\hline \multicolumn{1}{c}{ Variables } & $B$ & SE & LLCI & ULCI & $R^{2}(F)$ \\
\hline Conflicts perceived by adolescent children $\rightarrow$ Self-system traumatization & $.64^{* * *}$ & .07 & .51 & .77 & $.17\left(46.18^{* * *}\right)$ \\
Self-system traumatization $\rightarrow$ Bullying & $.07^{* * *}$ & .01 & .05 & .09 & $.19\left(35.46^{* * *}\right)$ \\
Conflicts perceived by adolescent children $\rightarrow$ Bullying & $.05^{* *}$ & .02 & .01 & .08 & .08 \\
Conflicts perceived by adolescent children $\rightarrow$ Bullying & $.09^{* * *}$ & .02 & .06 & .12 & $.12\left(32.45^{* * *}\right)$ \\
\hline
\end{tabular}

Note. $N=465$.

${ }^{* * *} p<.001 .{ }^{* *} p<.01$.

Table 3

Mediating Effects of Self-System Traumatization Bootstrapping Results

\begin{tabular}{lcccc} 
& & & & \multicolumn{2}{c}{ Boot } & Effect & Boot SE & LLCI & Boot ULCI \\
\hline Conflicts perceived by adolescent children $\rightarrow$ Self-system traumatization $\rightarrow$ Bullying & .04 & .01 & .03 & .06 \\
\hline
\end{tabular}

Note. $N=465$.

Table 4

Mediating Effects of Violence Toleranc in the Relationship Between Conflicts Perceived by Adolescent Children and Bullying

\begin{tabular}{lccccc}
\multicolumn{1}{c}{ Variables } & $B$ & SE & LLCI & ULCI & $R^{2}(F)$ \\
\hline Conflicts perceived by adolescent children $\rightarrow$ Violence tolerance & $.07^{* * *}$ & .01 & .06 & .10 & $.19\left(55.15^{* * *}\right)$ \\
Violence tolerance $\rightarrow$ Bullying & $.71^{* * *}$ & .07 & .58 & .84 & $.29\left(63.39^{* * *}\right)$ \\
Conflicts perceived by adolescent children $\rightarrow$ Bullying & $.04^{*}$ & .02 & .01 & .07 & .12 \\
Conflicts perceived by adolescent children $\rightarrow$ Bullying & $.09^{* * *}$ & .02 & .06 & $.12\left(32.45^{* * *}\right)$ \\
\hline
\end{tabular}

Note. $N=465$.

${ }^{* * *} p<.001 .{ }^{*} p<.05$.

마지막으로 폭력허용도는 또래괴롭힘 가해 $(r=.52, p<.001)$ 와 유의한 정적상관을 나타냈다. 전체 변인들의 상관관계 계 수는 .80 이하 $(r=.25 \sim .52)$ 이므로 다중공선성의 문제는 없는 것으로 나타났다.

\section{청소년 자녀가 지각한 부부갈등이 또래괴롭힘 가해에 미치는 영향에 대한 자기체계손상과 폭력 허용도의 개별매개효과}

\author{
청소년 자녀가 지각한 부부갈등이 또래괴롭힘 \\ 가해에 미치는 영향에 대한 자기체계손상의 매 \\ 개효과
}

청소년 자녀가 지각한 부부갈등이 또래괴롭힘 가해에 미치는 영향에 대한 자기체계손상의 매개효과 분석 결과는 Table 2 와 Table 3에 제시되었다. 먼저 Table 2의 경로별 회귀계수를 살펴 보면, 독립변인인 자녀가 지각한 부부갈등은 매개변인인 자기 체계손상 $(B=.64, p<.001)$ 에 유의한 정적영향을 미쳤고, 매개
변인인 자기체계손상은 종속변인인 또래괴롭힘 가해 $(B=.07$, $p<.001)$ 에 유의한 정적영향을 미치는 것으로 나타났다. 자녀 가 지각한 부부갈등이 또래괴롭힘 가해에 미치는 영향력은 매 개변인을 고려했을 때 감소했으며 통계적으로 유의한 것으로 나타나 $(B=.09, p<.001 \rightarrow B=.05, p<.01)$ 부분매개 모형이 지 지되었다. 매개효과가 통계적으로 유의한지를 확인하기 위해 부트스트래핑으로 간접효과를 검증한 결과(.03 .06), 95\% 신 뢰수준에서 신뢰구간의 값이 0 을 포함하지 않아 영가설이 기 각되었으므로 매개효과가 유의한 것으로 나타났다(Table 3).

\section{청소년 자녀가 지각한 부부갈등이 또래괴롭힘 가 해에 미치는 영향에 대한 폭력허용도의 매개효과}

청소년 자녀가 지각한 부부갈등이 또래괴롭힘 가해에 미치 는 영향에 대한 폭력허용도의 매개효과 분석 결과는 Table 4와 Table 5에 제시되었다. 먼저 Table 4의 경로별 회귀계수를 살펴 보면, 독립변인인 자녀가 지각한 부부갈등은 매개변인인 폭력 허용도 $(B=.07, p<.001)$ 에 유의한 정적영향을 미쳤고, 매개변 
Table 5

Mediating Effects of Violence Tolerance Bootstrapping Results

\begin{tabular}{ccccc}
\hline & & & \multicolumn{2}{c}{ Boot } \\
Variables & Effect & Boot SE & LLCI & Boot ULCI \\
\hline Conflicts perceived by adolescent children $\rightarrow$ Violence tolerance $\rightarrow$ Bullying & .05 & .01 & .04 & .08 \\
\hline
\end{tabular}

Note. $N=465$.

Table 6

Dual Mediating Effects of Self-System Traumatization and Violence Tolerance in the Relationship Between Conflicts Perceived by Adolescent Children and Bullying

\begin{tabular}{lccccc}
\hline \multicolumn{1}{c}{ Variables } & $B$ & SE & LLCI & ULCI \\
\hline Conflicts perceived by adolescent children $\rightarrow$ Self-system traumatization & $.64^{* * *}$ & .07 & .51 & .77 & $.17\left(46.18^{* * *}\right)$ \\
Conflicts perceived by adolescent children $\rightarrow$ Violence tolerance & $.06^{* * *}$ & .01 & .04 & .08 & $.21\left(40.85^{* * *}\right)$ \\
Self-system traumatization $\rightarrow$ Violence tolerance & $.02^{* *}$ & .01 & .01 & .04 & .04 \\
Self-system traumatization $\rightarrow$ Bullying & $.05^{* * *}$ & .01 & .03 & .07 & $.33\left(56.72^{* * *}\right)$ \\
Violence tolerance $\rightarrow$ Bullying & $.66^{* * *}$ & .07 & .53 & .79 & .07 \\
Conflicts perceived by adolescent children $\rightarrow$ Bullying & .01 & .02 & -.03 & .04 \\
Conflicts perceived by adolescent children $\rightarrow$ Bullying & $.09^{* * *}$ & .02 & .06 & .12 & $.12\left(32.45^{* * *}\right)$ \\
\hline
\end{tabular}

Note. $N=465$.

${ }^{* * *} p<.001 .{ }^{* *} p<.01$.

인인 폭력허용도는 종속변인인 또래괴롭힘 가해 $(B=.71, p<$ .001)에 유의한 정적영향을 미치는 것으로 나타났다. 자녀가 지각한 부부갈등이 또래괴롭힘 가해에 미치는 영향력은 매개 변인을 고려했을 때 감소했으며 통계적으로 유의한 것으로 나 타나 $(B=.09, p<.001 \rightarrow B=.04, p<.05)$ 부분매개 모형이 지 지되었다. 매개효과가 통계적으로 유의한지를 확인하기 위해 부트스트래핑으로 간접효과를 검증한 결과(.04 .08), 95\% 신 뢰수준에서 신뢰구간의 값이 0을 포함하지 않아 영가설이 기 각되었으므로 매개효과가 유의한 것으로 나타났다(Table 5).

\section{청소년 자녀가 지각한 부부갈등이 또래괴롭힘 가해에 미치는 영향에 대한 자기체계손상과 폭력 허용도의 이중매개효과}

먼저 Table 6의 경로별 회귀계수를 살펴보면, 독립변인인 자 녀가 지각한 부부갈등은 제 1 의 매개변인인 자기체계손상 $(B=$ $.64, p<.001)$ 과 제 2 의 매개변인인 폭력허용도 $(B=.06, p<.001)$ 에 각각 유의미한 정적영향을 미치는 것으로 나타났다. 또한 제 1 매개변인인 자기체계손상은 제 2 매개변인인 폭력허용도 $(B$ $=.02, p<.01)$ 와 종속변인인 또래괴롭힘 가해 $(B=.05, p<.001)$ 에 유의한 정적영향을 미치고, 폭력허용도는 또래괴롭힘 가해 $(B=.66, p<.001)$ 에 유의한 정적영향을 미치는 것으로 나타났 다. 자녀가 지각한 부부갈등과 또래괴롭힘 가해와의 관계에서
자기체계손상과 폭력허용도를 매개했을 때, 자녀가 지각한 부 부갈등이 또래괴롭힘 가해에 미치는 직접적인 영향력은 유의 하지 않아 자기체계손상과 폭력허용도가 두 변인간의 관계를 완전매개하는 것으로 나타났다. 결과적으로, 자녀가 지각한 부 부갈등은 자기체계손상과 폭력허용도라는 매개를 거쳐 최종 적으로 또래괴롭힘 가해에 영향을 미치는 요인으로 작용함을 알 수 있다. 이러한 이중매개효과가 통계적으로 유의한지 확 인하기 위해 부트스트래핑 방법으로 간접효과를 검증한 결과 (.01 .06), 95\% 신뢰수준에서 신뢰구간의 값이 0을 포함하지 않아 영가설이 기각되었으므로 이중매개 효과가 유의한 것으 로 나타났다. 또한 자녀가 지각한 부부갈등과 또래괴롭힘 가해 와의 관계에서 자기체계손상(.02 .05)과 폭력허용도(.02 .06) 의 개별 매개효과 역시 각각 유의한 것으로 나타났다(Table 7). 이와 같은 결과를 도식화한 최종 모형은 Figure 2와 같다.

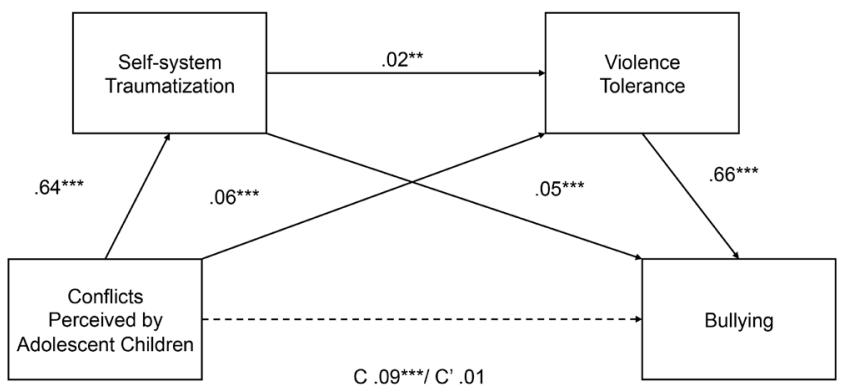

Figure 2. Final research model. 
Table 7

Dual Mediating Effects of Self-System Traumatization and Violence Tolerance on Bootstrapping

\begin{tabular}{|c|c|c|c|c|}
\hline & & Boot & Boot & Boot \\
\hline & Effect & SE & LLCI & ULCI \\
\hline Total Effect & .08 & .01 & .06 & 11 \\
\hline Conflicts perceived by adolescent children $\rightarrow$ Self-system traumatization $\rightarrow$ Bullying & .03 & .01 & .02 & .05 \\
\hline Conflicts perceived by adolescent children $\rightarrow$ Violence tolerance $\rightarrow$ Bullying & .04 & .01 & .02 & .06 \\
\hline Conflicts perceived by adolescent children $\rightarrow$ Self-system traumatization $\rightarrow$ Violence tolerance $\rightarrow$ Bullying & .01 & .01 & .01 & .02 \\
\hline
\end{tabular}

Note. $N=465$.

\section{Discussion}

본 연구는 청소년 자녀가 지각하는 부부갈등과 또래괴롭힘 가 해와의 관계에서 자기체계손상과 폭력허용도의 매개효과를 알아보고자 하였다. 이를 통해 또래괴롭힘 가해 청소년 발생 률을 줄이기 위하여 가정 안에서 부모의 역할과 상담 시 개입 방법을 탐색하였다. 연구 결과를 보면 다음과 같다.

첫째, 청소년 자녀가 지각한 부부갈등이 또래괴롭힘 가해 에 미치는 영향에 대해 자기체계손상과 폭력허용도는 각각 부 분 매개효과를 갖는 것으로 확인되었다. 이는 자녀가 지각한 부부갈등이 또래괴롭힘 가해에 직접 영향을 미치거나 자기체 계손상 또는 폭력허용도라는 매개변인을 통해 간접적으로 또 래괴롭힘 가해에 영향을 미칠 수 있음을 가리킨다. 즉, 자녀가 지각한 부부갈등이 자기체계손상과 폭력허용도를 증가시킴 으로써 또래괴롭힘 가해에 영향을 미치는 것이다.

먼저 자녀가 지각한 부부갈등이 자기체계손상이라는 매개 변인을 통해 간접적으로 또래괴롭힘 가해에 영향을 미친다는 결과는 부부갈등과 같은 대인간 외상을 경험한 자녀들이 주 양육자와 부정적인 상호작용을 하면서 자기(self)가 형성되기 보다는 손상되며(J. Jang, 2010), 대인관계문제를 일으킨다는 선행연구를 통해 지지된다(H.-R. Kim \& Hong, 2017; Wilson, Droždek, \& Turkvoic, 2006). 이는 대상적 자기손상으로 낮은 자존감을 갖게 된 청소년이 또래괴롭힘을 일으키는 기제로 작 용함을 의미한다(H.-Y. Kwon \& Kim, 2009; M.-H. Lee \& Yoo, 2005). 자존감이 낮은 청소년들은 자신을 낮게 평가하는 사회 적 경험을 피하고 자신의 자존감을 회복하기 위하여 보상적 인 수단으로 비행행위를 한다는 선행연구의 주장과 맥락을 같이한다(H.-Y. Kwon \& Kim, 2009; Rosenberg, Rosenberg, \& $\mathrm{McCord}, 1978)$. 또한, 자기조절손상으로 자신의 정서.행동.충 동 조절이 어렵게 되면 또래괴롭힘을 일으킬 가능성이 높아지 는데 이것은 또래괴롭힘 가해청소년들이 폭력행동에 대해 정 서적으로 무감각하다는 것과 정서지각이 낮을 수 있다는 선행
연구로 뒷받침되며(D. G. Kim, Jang, \& Han, 2017; J. E. Oh \& $\mathrm{Kim}, 2017)$, 또래괴롭힘 가해 청소년들이 주도적 폭력보다 충 동조절 어려움으로 일어나는 반응적 폭력행동을 더 많이 한 다는 선행연구에 의해 지지된다(J.-E. Oh \& Lee, 2014). 이 결 과들을 토대로 또래괴롭힘 가해청소년에게 치료적 개입을 할 때, 부모의 부부갈등 여부를 살피고 가해 청소년의 낮은 자존 감, 불명확한 자기개념, 수치심과 공격성, 정서.행동·충동조절 의 어려움으로 드러나는 손상된 자기 체계에 초점을 맞추는 것이 필요함을 알 수 있다.

최근 Fonagy와 Lyuten (2009)은 자기-관련 병리를 멘탈라이 제이션(mentalization)과 같은 반영능력(reflection)의 결함으로 보는 견해에 따라 멘탈라이제이션을 기반으로 한 Creating a Peaceful School Learning Environment (CAPSLE)를 실시하여 학 생들의 공격성과 문제행동이 감소하는 효과를 가져왔다. 멘 탈라이제이션은 어린 시절 부모의 반영을 통해 발달하게 되는 메타인지 중 하나인 상위 정서조절능력으로(Allen \& Fonagy, 2002), 자신의 정서를 명확히 인식·조절하고 타인의 정서를 인 식하고 이해할 수 있는 능력이라고 할 수 있다. 이와 관련하 여, 정서를 인식하고 조절하는 심리치료로 Cognitive Behavioral Therapy (CBT)가 효과적이므로 CBT를 활용하여 자신의 생각 과 감정을 연결시키는 작업을 통해 감정을 명확하게 인식하고 조절하며, 나아가 타인의 감정을 공감하는데 도움이 될 것이다. 자녀가 지각한 부부갈등이 폭력허용도라는 매개변인을 통 해 간접적으로 또래괴롭힘 가해에 영향을 미친다는 결과는 가정에서 부부폭력과 같은 정서적 학대에 노출된 자녀가 폭 력에 대해 허용적인 태도를 보이며(K.-H. Noh \& Hahn, 2017; J.-A. Oh et al., 2016), 폭력에 대해 허용적인 태도가 또래괴롭 힘 가해에 영향을 미친다는 연구와 같은 맥락으로 볼 수 있다 (K.-E. Kim \& Yoon, 2012; J.-Y. Kim et al, 2015). 또한 아동기에 신체 및 심리적 학대를 당한 사람이 청소년기에 폭력의 가해 자가 될 가능성이 높다는 선행연구에 의해 지지된다(Mun \& Chung, 2015; J. A. Oh \& Kim, 2015). 
자녀들이 부모의 부부갈등을 많이 경험할수록 폭력에 대 한 허용도가 높아지며 실제 또래괴롭힘이 발생할 확률이 높아 진다는 것을 알 수 있다. 이는 부부갈등과 같은 부모 간 폭력을 직.간접적으로 경험한 또래괴롭힘 가해청소년들은 폭력에 대 한 잘못된 개념을 학습하여 폭력행동을 한다고 볼 수 있다. 또 래괴롭힘 가해 청소년을 상담하는데 있어 폭력에 대한 허용적 태도에 초점을 맞추고 폭력에 대한 잘못된 이해를 변화시키는 데 집중하여 상담 전략을 수립하는 것이 효과적일 수 있다. 이 들이 가지고 있는 갈등상황에서 폭력 사용이 유용하다는 비합 리적 신념을 파악하고 인지적 재구성(cognitive reconstruction) 을 통해 폭력 사용에 대한 사고의 변화를 기대할 수 있을 것이 다. 또한 스스로의 생각과 정서와 행동에 대한 객관적인 자기 감찰훈련(self-monitoring)을 병행하는 사고변화기록지(thought change records)을 통하여 자신의 감정을 명확히 인식하고 충동 적인 행동을 조절하도록 하는 것이 도움이 될 것이다.

둘째, 청소년 자녀가 지각한 부부갈등이 또래괴롭힘 가해 에 미치는 영향에 대해 자기체계손상과 폭력허용도는 완전 이 중매개효과를 갖는 것으로 밝혀졌다. 자녀가 지각한 부부갈등 이 높을수록 자기체계손상이 높아지고, 높아진 자기체계손상 은 폭력허용도를 높여서 또래괴롭힘 가해를 증가시킨다. 자녀 가 지각한 부부갈등은 이중매개 관계에서 직접적으로 또래괴 롭힘 가해에 미치는 영향력이 사라지고, 자기체계손상과 폭력 허용도를 통해서 영향을 미치는 것으로 나타났다. 따라서, 또 래괴롭힘 가해 청소년을 대상으로 치료를 계획할 때 부모의 부부갈등 해결방법에 대한 부모교육이 진행되는 동시에 가해 청소년의 손상된 자기체계와 폭력을 허용하는 인식을 우선적 으로 고려해서 치료계획을 세우는 것이 효과적이라고 할 수 있다.

자존감이 높을수록 폭력허용도가 낮아지고 자존감이 낮을 수록 폭력허용도가 높아진다는 선행연구(J.-Y. Ahn, 2016; B.-S. Lee, 2007)는 자기체계가 손상되어 폭력허용도가 높아진다는 본 연구의 내용을 지지한다. 이는 대상적 자기가 손상되어 낮 은 자기 가치감을 가진 청소년들이 자신이 폭력을 당해도 괜찮 다고 생각하거나 자신의 낮은 가치감의 회복을 위해 폭력을 사 용하는 것에 대한 허용정도가 높아지게 되는 것으로 보인다.

또한 자기체계 손상의 하위요인인 자기조절손상이 높을수 록 폭력허용도도 높아진다는 연구결과는 자신과 타인의 정서 를 효율적으로 인식·표현·조절하는 능력인 정서지능과 공감능 력이 낮을수록 학교폭력에 대해 허용적이었으며(H. Y. Kang $\& \mathrm{Kim}, 2014 ;$ J.-O. Kim \& Kim, 2019) 공감과 같은 정서조절 능력이 뛰어날수록 폭력허용도가 낮다는 선행연구에 의해 지
지된다(J.-I. Lee \& Kim, 2015). 이는 자기체계손상은 자신의 정서.행동·충동성 조절을 어렵게 하며 사소한 갈등상황이나 작은 자극에도 폭력에 대한 허용정도를 높여서 또래괴롭힘을 일으키는 기제로 작용하는 것이다.

본 연구는 자녀가 지각한 부부갈등이 또래괴롭힘 가해에 미치는 영향에서 자기체계손상과 폭력허용도의 이중매개 역 할을 검증함으로 개념들의 관계성을 명확히 밝혀냈다는데 의 의가 있다. 기존의 또래괴롭힘 가해 연구에서는 자존감이나 공격성, 정서조절, 자기통제와 같은 부분에 대한 연구가 진행 되긴 했지만 총체적인 자기개념에 대해 살펴본 연구는 없었 다. 또한, 자녀가 지각한 부부갈등, 자기체계손상, 폭력허용도, 또래괴롭힘 가해에 대한 연구도 단편적인 관계검증에 머물렀 다. 이에 본 연구는 자녀가 지각한 부부갈등이 또래괴롭힘 가 해를 일으키는 과정에서 단순히 폭력에 대한 허용도가 높아져 서 가해를 일으키는 것이 아니라, 자기체계손상이 되었기 때 문에 폭력 허용도가 높아지고 이것이 또래괴롭힘 가해를 일으 킨다는 것을 확인하였다. 이와 같은 결과는 또래괴롭힘 가해 청소년들을 위한 개입으로 일회성 상담과 봉사활동과 같은 단 순한 개입뿐만이 아니라 개인 심리구조의 변화, 더 구체적으 로 주체적 자기.대상적 자기.자기조절·관계적 자기를 포함하 는 다차원적인 자기체계의 변화를 살펴보고 이를 적용하여 치 료적 개입을 시도하는 것이 효과적인 치료방향이 될 것으로 보인다. 종합하면 본 연구는 자녀가 지각한 부부갈등이 또래 괴롭힘 가해에 이르는 경로를 밝히는 데 기여했으며, 이를 통 해 또래괴롭힘 가해 청소년들을 대상으로 한 심리치료 개입 및 프로그램 개발에 유용한 기초 자료를 제공했다는 데 의의 가 있다.

본 연구가 갖는 제한점과 후속 연구를 위한 제언은 다음과 같다.

첫째, 서울 및 수도권에 위치한 중·고등학교 학생이라는 제 한된 표본을 대상으로 분석하였기에 국내 전체 남녀 청소년에 게 연구결과를 일반화 할 수 없다는 점이다. 이후 연구에서는 성별, 연령, 지역 등을 고려하여 다양한 표집을 통해 연구의 대 표성과 일반화 가능성을 높여야 할 것이다.

둘째, 본 연구는 자기보고식 척도를 사용하여 변인들을 측 정하였다는 점에서 한계를 가진다. 자기보고식 척도를 사용할 경우 자신의 경험을 축소.확대할 수 있으며, 자신을 긍정적으 로 보이고자하는 사회적 바람직성이 있어 자료의 신뢰성이 떨 어질 수 있다. 특히, 사회적으로 부정적인 인식이 있는 부부갈 등, 폭력허용도, 또래괴롭힘 가해에 대한 자기보고식 척도는 자신의 경험을 축소할 가능성이 높다. 그러므로 이런 문제를 
해결하기 위해서 후속연구에서는 제 3 자에 의한 보고나 또래 괴롭힘 가해 청소년을 대상으로 질적 연구도 고려해야 할 필 요성이 있다.

셋째, 본 연구의 주요 변인인 또래괴롭힘 가해, 자녀가 지각 한 부부갈등, 자기체계손상 및 폭력허용도는 장기적인 태도와 인식으로 형성된 것으로 본 연구에서 횡단연구가 진행되었다 는 한계가 있다. 주요 변인 속성을 기반으로 종단적 연구가 추 가적으로 진행되는 것 역시 의미가 있을 것이다.

마지막으로, 본 연구에서는 자녀가 지각한 부부갈등으로 인한 또래괴롭힘 가해를 중점으로 살펴보았으나 또래괴롭힘 의 피해와 가해 중복 경험자에 대해서도 연구가 필요하다. 최 근의 연구에 따르면 또래괴롭힘 피해를 경험한 청소년들이 가 해 학생에게 복수하고 싶은 충동 때문에 자신보다 약한 학생 을 괴롭히는 현상이 일어나고 있다(Ah \& Jeong, 2012; Pranjic $\&$ Bajraktarevic, 2010). 이렇듯 또래괴롭힘 피해를 경험한 청소 년들이 피해자이면서 가해자의 위험에 노출되어 있으므로 또 래괴롭힘 피해·가해 중복경험자 대한 후속 연구가 필요하다.

\section{Notes}

This article is based on a part of the first author's master's thesis from Myongji University (2019), and this article was presented at the 2019 Annual Spring Conference of the Korean Association of Child Studies, Seoul, Korea.

\section{Conflict of Interest}

No potential conflict of interest relevant to this article was reported.

\section{References}

\section{In English}

Allen, J. G. (2001). Traumatic relationships and serious mental disorders. Hoboken, NJ: Wiley.

Allen, J. G., \& Fonagy, P. (2002). The development of mentalizing and its role in psychopathology and psychotherapy. Topeka, KS: Meninger Clinic.

Baldry, A. C., \& Farrington, D. P. (2000). Bullies and delinquents: Personal characteristics and parental styles. Journal of Community \& Applied Social Psychology, 10(1), 17-31. doi:
10.1002/(SICI) 1099-1298(200001/02)10:1<17::AIDCASP526>3.0.CO;2-M

Boivin, S., Lavoie, F., Hébert, M., \& Gagne, M. H. (2012). Past victimizations and dating violence perpetration in adolescence: The mediating role of emotional distress and hostility. Journal of Interpersonal Violence, 27(4), 662-684. doi:10.1177/0886260511423245

Buchanan, C. M., \& Waizenhofer, R. (2001). The impact of interparental conflict on adolescent children: Considerations of family systems and family structure. In A. Booth, A. C. Crouter, M. Clements (Eds.), Couples in conflict (pp. 149160). London: Routledge.

Buehler, C., \& Gerard, J. M. (2002). Marital conflict ineffective parenting, and children's and adolescents' maladjustment. Journal of Marriage and Family, 64(1), 78-92. doi:10.1111/ j.1741-3737.2002.00078.x

Collins, W. A., \& Laursen, B. (2004). Changing relationships, changing youth: Interpersonal contexts of adolescent development. The Journal of Early Adolescence, 24(1), 5562. doi:10.1177/0272431603260882

Cook, A., Spinazzola, J., Ford, J., Lanktree, C., Blaustein, M., Cloitre, M.,...Van der Kolk, B. (2005). Complex trauma in children and adolescents. Psychiatric Annals, 35(5), 390-398. doi:10.3928/00485713-20050501-05

Fincham, F. D., Grych, J. H., \& Osborne, L. N. (1994). Does marital conflict cause child maladjustment? Directions and challenges for longitudinal research. Journal of Family Psychology, 8(2), 128-140. doi:10.1037/0893-3200.8.2.128

Finkel, E. J., \& Baumeister, R. F. (2010). Advanced social psychology: The state of the science (2nd ed.). London: Oxford University Press.

Fonagy, P., \& Luyten, P. (2009). A developmental, mentalization-based approach to the understanding and treatment of borderline personality disorder. Development and Psychopathology, 21(4), 1355-1381. doi:10.1017/s0954579409990198

Gini, G., \& Espelage, D. L. (2014). Peer victimization, cyberbullying, and suicide risk in children and adolescents. Jama, 312(5), 545-546. doi:10.1001/jama.2014.3212

Grych, J. H., Seid, M., \& Fincham, F. D. (1992). Assessing marital conflict from the child's perspective: The children's perception of inter parental conflict Scale. Child Development, 63(3), 558-572. doi: $10.2307 / 1131346$

Hanish, L. D., \& Guerra, N. G. (2002). A longitudinal analysis of patterns of adjustment following peer victimization. Development and psychopathology, 14(1), 69-89. doi:10.1017/ s0954579402001049

Harter, S. (1999). The construction of the self: A developmental perspective. New York: Guilford Press.

Hayes, A. F. (2013). Introduction to mediation, moderation, and conditional process analysis: A regression-based approach. New 
York: Guilford Press.

Kline, R. B. (2010). Principles and practice of structural equation modeling. New York: Guilford Press.

Olweus, D. (1978). Aggression in the schools: Bullies and whipping boys. Washington, DC: American Psychological Association.

Olweus, D. (1993). Bullying at school. Hoboken, NJ: Wiley Blacwell.

Ozer, E. J., \& Weinstein, R. S. (2004). Urban adolescents' exposure to community violence: The role of support, school safety, and social constraints in a school-based sample of boys and girls. Journal of Clinical Child and Adolescent Psychology, 33(3), 463-476. doi:10.1207/s15374424jccp3303_4

Perry, D. G., Kusel. S. J., \& Perry, L. C. (1988). Victims of peer aggression. Developmental Psychology. 24(6), 807-814.

Perry, D. G., Perry, L. C., \& Ramussen, P. (1986). Cognitive social learning mediators of aggression. Child Development, 57(3), 700-711. doi:10.1111/j.1467-8624.1986.tb00238.x

Pranjić, N., \& Bajraktarević, A. (2010). Depression and suicide ideation among secondary school adolescents involved in school bullying. Primary Health Care Research \& Development, 11(4), 349-362. doi:10.1017/s1463423610000307

Rigby, K., \& Slee, P. T. (1991). Bullying among Australian school children: Reported behavior and attitudes toward victims. The Journal of Social Psychology, 131(5), 615-627. doi:10.10 80/00224545.1991.9924646

Rosenberg, F. R., Rosenberg, M., \& McCord, J. (1978). Selfesteem and delinquency. Journal of Youth and Adolescence, 7(3), 279-294. doi:10.1007/bf01537978

Salo, J., Punamäki, R. L., \& Qouta, S. (2004). Associations between self and other representations and posttraumatic adjustment among political prisoners. Anxiety, Stress \& Coping, 17(4), 421-440. doi:10.1080/1061580031000075814

Swearer, S. M., Song, S. Y., Cary, P. T., Eagle, J. W., \& Mickelson, W. T. (2001). Psychosocial correlates in bullying and victimization: The relationship between depression, anxiety, and bully/victim status. Journal of Emotional Abuse, 2(2-3), 95-121. doi:10.1300/j135v02n02_07

Van der Kolk, B. A., Roth, S., Pelcovitz, D., Sunday, S., \& Spinazzola, J. (2005). Disorders of extreme stress: The empirical foundation of a complex adaptation to trauma. Journal of Traumatic Stress, 18(5), 389-399. doi:10.1002/jts.20047

Wilson, J. P., Droždek, B., \& Turkovic, S. (2006). Posttraumatic shame and guilt. Trauma, Violence, \& Abuse, 7(2), 122-141. doi:10.1177/1524838005285914

\section{In Korean}

Ah, Y.-A., \& Jeong, W.-C. (2012). Mediating effects of internet addiction in the relationship between school violence aggression and victimization. Korean Journal of Youth
Studies, 19(12), 331-354.

Ahn, J.-M., \& Ahn, K.-S. (2012). A study on related factors for adolescent's anger emotional experience. Korean Journal of Youth Studies, 19(3), 267-292.

Ahn, J.-Y. (2016). The effect of elementary school children's degree of violence acceptance, empathy and ego-resilience on the experience of school violence. Asia-pacific Journal of Multimedia Services Convergent with Art, Humanities, and Sociology, 6, 175-183.

Cha, J., Lee, J., \& Hwang, S. (2009). The mediating effect of shame and fear of abandonment on adolescents' depression and aggression : Comparison of boy and girl students model. Journal of Youth Facilities and Environment, 7(2), 3-15.

Cho, C. B., \& Cho, N. H. (2011). The effect of exposed experience of adolescents to family violence on committing school violence: Focused on the mediating effect of self-control. Korean Journal of Youth Studies, 18(4), 75-102.

Choi, E. S. (2000). A study of psychological factors associated with bullying (Master's thesis). Retrieved from http://www.riss. $\mathrm{kr} / \mathrm{link}$ ?id=T8422244

Choi, H.-Y. (2011). The effects of NIE(Newspaper In Education) program on the aggression and violence tolerance of school violence adolescent offenders (Master's thesis). Retrieved from http://www.riss.kr/link?id=T12382013

Hur, S.-H., \& Nam, S.-K. (2016). Effects of family functioning, marital conflicts, and experiences of exposure to violence on aggression. Social Science Review, 34(2), 247-270.

Im, J.-I., \& Kim, Y.-S. (2010). Levels of acceptance of violence by parents and the effects on their children's experiences of bullying. The Korean Journal of Human Development, 17(1), 243-262.

Im, J.-W., \& Hong, H.-Y. (2016). The influence of internalized shame on the relational aggression of college students-the mediating effects of dysfunctional anger expression. Journal of the Korea Institute of Youth Facilities and Environment, 14(1), 63-75.

Im, S. I., \& Lee, J. M. (2013). Relationship between witness of inter-parents violence, self-esteem, victims and bullies of school violence in male high school students. Journal of School Social Work, 26, 27-45.

Jang, J. (2010). Traumatized self-system in adults repetitively exposed to interpersonal trauma (Doctoral dissertation). Retrieved from http://www.riss.kr/link?id=T12115177

Jang, J. A., \& Shin, H. C. (2006). The influence of marital conflict on adolescent's maladaptation: Mediating effects of ego-resiliency, parent's child-rearing attitudes and social support. The Korean Journal of Counseling And Psychotherapy, 18(3), 569-592.

Kang H. Y., \& Kim, Y. H. (2014). Effect of emotional intelligence on the school violence attitude and latent delinquency of adolescents. Korean Juvenile Protection Review, 24, 31-59.

Kim, A., \& Lee, S. (2016). The moderating effects of self-esteem 
in children's perceived interparental conflict and depressive symptoms. Korean Journal of Play Therapy, 19(3), 211-226. doi:10.17641/kapt.19.3.2

Kim, D. G., Jang, S. E., \& Han, H. W. (2017). The mediated effects of emotion regulation in the relations between mother attachment and school violence of elementary school students. Journal of Learner-Centered Curriculum and Instruction, 17, 751-772. doi:10.22251/jlcci.2017.17.17.751

Kim, H. (2013). An effect of persistent school violence victimization experience on youths' physical, socioemotional, and cognitive development. Journal of Adolescent Welfare, 15(2), 121-143.

Kim, H.-R., \& Hong, H.-Y. (2017). The influence of emotional abuse in childhood on dysfunctional anger expression of university students: The mediating effects of traumatized selfsystem and internalized shame. Korean Journal of Counseling, 18(6), 321-340. doi:10.15703/kjc.18.6.201712.321

Kim, I. S. (2018, October 05). Wigiui haggyo... haggyopoglyeog jibdangahaedo, jasal wiheom hagsaengdo 3nyeon sae 2bae $\uparrow$ [위 기의 학교... 학교폭력 집단가해도, 자살 위험 학생도 3 년 새 2배 $\uparrow$. Hangugilbo Retrieved from http://www.hankookilbo. $\mathrm{com} / \mathrm{News} / \operatorname{Read} / 201810051410364686$ ?did=na

Kim, J. (2011). The effect of perceived marital conflicts on psychosocial maladjustment. : The mediating role of parentification (Master's thesis). Retrieved from http://www.riss.kr/ link?id=T12281347

Kim, J. Y., Choi, S. A., \& Lim, J. H. (2015). Moderating effects of acceptability of violence on the impact of community environments on adolescents' school violence. Korean Journal of Youth Studies, 22(11), 111-135.

Kim, J. Y., Jang, Y. E., \& Min, J. A. (2011). A study on the effect of school violence to adolescent's school adjustment: Moderating effect of parent-child commuinication. Korean Journal of Youth Studies, 18(7), 209-234.

Kim, J.-H., \& Lee, S. (2017). The mediating effects of adolescents' aggressive behaviors on the relationship between perceived parents' marital conflict and school adjustment. Korean Journal of Play Therapy, 20(3), 181-199.

Kim, J-O., \& Kim, W-I. (2019). The mediating effects of class cohesiveness in the relationship between empathic ability and school violence attitude of upper grade students in elementary school. Korean Journal of Youth Studies, 26(1), 205-226. doi:10.21509/kjys.2019.01.26.1.205

Kim, K., Lee, Y., \& Shin, J. (2013). A study on the perceptual differences of school communities in the realities and causes of school violence. The Journal of Yeolin Education, 21(4), 95-120.

Kim, K.-E., \& Yoon, H. (2012). Associations between adolescents' victimization of violence, tolerance toward violence, and cyber violence offending behavior. Journal of The Korean Society of Child Welfare, 39, 213-244.
Kim, Y. S., Park, J. S., \& Kim, Y. H. (2018). A longitudinal study on the effect of parenting marital conflict and parenting depression on internalization and externalization problem behaviors of children. Journal of Future Oriented Youth Society, 15(1), 135-155. doi:10.34244/fy.2018.15.1.135

Kim, Y.-S. (2000). Influencing factors on bullying of elementary school pupils (Master's thesis). Retrieved from http://www.riss.kr/ link?id=T7646791

Kwon, H.-Y., \& Kim, H.-M. (2009). The qualitative analysis on psycho-social factors of bullying adolescent at school violence. The Korean Journal of East West Science, 12(1), 1-12.

Kwon, J. K. (2011). The developmental trajectories of victimized experiences and the changing behavior problems-a longitudinal study with applied LCGA \& GMM with known Class-. Journal of the Korean Society of Child Welfare, 34, 95-126.

Kwon, Y. O., \& Lee, C. D. (1997). A validation study on the children's perception of interparental conflict scale. Korean Journal of Child Studies 18(1), 65-78.

Lee, B.-S. (2007). The effects of parenting behavior on self-esteem, aggression, school violence experience, violence tolerance as perceiveed by adolescence. Journal of School Social Work, 13, 25-50.

Lee, C.-J. \& Kwak, K.-J. (2000). Haggyoeseoui jibdanttadollim: Siltaewa teugseong. [학교에서의 집단따돌림: 실태와 특 성]. Seoul: Jimmundang.

Lee, J.-I., \& Kim, C.-D. (2015). The effects of empathic perspective-taking on aggression and permissive attitudes towards bullying in elementary school children. Korea Journal of Counseling, 16(5), 253-266. doi:10.15703/ kjc. 16.5.201510.253

Lee, M.-H., \& Yoo, S.-H. (2005). Differences in ego-identity status and social problem solving ability by experience in committing school violence. Journal of Educational Research, 15, 51-68.

Lee, S. W., \& Jo, S. S. (2005). A study on the effects of violence experience and attitudes on wife battering. Korean Journal of Social Welfare Research, 13, 111-131.

Lee, S. Y., \& Jung, H. G. (2015). On school violence behavior within communication skills. Journal of Social Welfare Management, 2(2), 83-103.

Min, D. K., \& Choi, M.-K. (2014). The influence of parents conflict on youth's anxiety and school adaptation. Journal of the Korean Data \& Information Science Society, 25(6), 1407 1418. doi:10.7465/jkdi.2014.25.6.1407

Ministry of Education. (2018). 2018nyeon 1cha hakgyopongnyeok siltaejosa gyeolgwa [2018년 1차 학교폭력 실태조사 결과] Retrieved from MOE Blog: https://blog.naver.com/ moeblog/221346805834

Moon, C.-H., Park, J., Jeon, J.-A., \& Cheon, S.-M. (2010). The effect of husband-and-wife conflicts on peer-relationships among juveniles -by the medium of self-perception and 
depression-. The Journal of Humanities, 15(1), 255-273.

Moon, Y.-L., Choi, J., Paek, S., \& Kim, Y.-J. (2007). Gender difference in the occurrence of school violence: Analyzing victim 's counseling cases. The Korean Journal of Educational Psychology, 21(3), 703-722.

Mun, J.-H., \& Chung, H. (2015). College students' experiences of dating violence and violence tolerance. Family and Family Therapy, 23(4), 627-653. doi:10.21479/kaft.2015.23.4.627

Noh, K.-H., \& Hahn, D.-D. (2017). The effects parenting attitude on aggression and sense of school community perceived by elementary school students. Journal of Educational Innovation Research, 27, 159-180. doi:10.21024/pnuedi.27.1.201703.159

Noh, K.-H., \& Joo, C.-A. (2015). The effects of homeroom teacher's emotional leadership on school violence allowance and sense of school community perceived by elementary school students. The Journal of Educational Administration, 33, 151-171.

Oh, J. A., \& Kim, Y. H. (2015). The effects of inter-parental conflict and parental behavior on self-perception and school adjustment: Focused on gender difference of adolescents in multi-cultural family. Korean Journal of Youth Studies, 22(6), 205-231.

Oh, J. A., Kim, E. K., \& Yoon, H. (2018). Effects of parental conflict and teacher behavior on adolescents' psychological adaptation and bully/victim status. Journal of School Social Work, 41, 221-246. doi:10.20993/jssw.41.10

Oh, J. E., \& Kim, S. Y. (2017). The relationship between early adolescents' emotional intelligence, aggression, school violence perception and coping attitude. Journal of School Social Work, 40, 195-215. doi:10.20993/jssw.40.9

Oh, J.-A., Park, J.-H., \& Lim, S.-J. (2016). The influence of parental conflict, the father's parenting behavior, and violence acceptability on the perpetration of school violence. Korean Journal of Family Welfare, 21(3), 565-582. doi:10.13049/kfwa.2016.21.3.9

Oh, J.-E., \& Lee, J.-Y. (2014). Mediating effects of anger expression types for anxiety on the violent behaviors of adolescent school violence offenders and general adolescents. Journal of Korean Home Management Association, 32(5), 163-178. doi:10.7466/jkhma.2014.32.5.163

Oh, M. K,. Ahn, J. Y., \& Kim, J. S. (2013). The effect of children's sensation seeking on experience of committing a school violence: Moderating effect of communication with parents. Korean Journal of Youth Studies, 20(3), 31-54.

Park, M.-J., \& Kang, J.-H. (2017). The effect of the adolescents' perception of inter-parental conflict on the depression and aggression: Moderating effects of self-compassion. Korea Journal of Counseling, 18(4), 167-190. doi:10.15703/ kjc.18.4.201708.167

Seol, K. O., Kyeong, Y., \& Ji, Y. J. (2015). The effects of adolescent shyness, self-regulation, and gender on peer victimization : Tests of a three-way interaction. The Korean Journal of Developmental Psychology, 28(4), 33-54.

Shin, S. C., \& Lee, J. C. (2015). Double mediation estimates of internalized shame and aggression with regard to effects of excessive use of a smart phone by schoolchildren on youth violent behavior. Journal of Koran Society for Youth Protection and Guidance, 22, 89-108.

Song, E.-J., \& Lee, J.-Y. (2010). The effect of marital conflicts on youth depression-with the mediation of parentification. Korea Journal of Counseling, 11(1), 335-354. doi:10.15703/ kjc.11.1.201003.335

Song, H. S. (2014). The effect of domestic violence, school violence and depression-anxiety on adolescent suicide (Doctoral dissertation). Retrieved from http://www.riss.kr/link?id= T13534501

Won, M.-J., \& Gong, J. Y. (2017). The mediation effect of parentchild communication on the relationship between children's perceived marital conflict and self-esteem. The Korean Journal of Elementary Counseling, 16(3), 285-299.

Yoon, M. S., Lee, M. S., \& Min, S. Y. (2012). The mediating effects of depression on the relationship of parental conflict exposure and school violence. Journal of Adolescent Welfare, 14(4), 237-259.

\section{ORCID}

Su Jeong Kim https://orcid.org/0000-0002-1350-8562

Jung Min Kim http://orcid.org/0000-0002-6611-6212

In Hee Hwang https://orcid.org/0000-0003-0448-7963

Received October 31, 2019

Revision received November 24, 2019

Accepted December 15, 2019 